Universal States of Finite Anti-Plane Shear: Ericksen's Problem in Miniature Author(s): James K. Knowles

Source: The American Mathematical Monthly, Vol. 86, No. 2 (Feb., 1979), pp. 109-113

Published by: Mathematical Association of America

Stable URL: http://www.jstor.org/stable/2321944

Accessed: 20/10/2014 19:33

Your use of the JSTOR archive indicates your acceptance of the Terms \& Conditions of Use, available at http://www.jstor.org/page/info/about/policies/terms.jsp

JSTOR is a not-for-profit service that helps scholars, researchers, and students discover, use, and build upon a wide range of content in a trusted digital archive. We use information technology and tools to increase productivity and facilitate new forms of scholarship. For more information about JSTOR, please contact support@ jstor.org. 


\section{UNIVERSAL STATES OF FINITE ANTI-PLANE SHEAR: ERICKSEN'S PROBLEM IN MINIATURE}

\section{JAMES K. KNOWLES}

1. Introduction. A solid body which in its undeformed state occupies a cylindrical region is said to undergo a deformation corresponding to anti-plane shear if each particle of the body is displaced parallel to the generators of the cylinder by an amount which is independent of the axial position of the particle. The displacement vector field thus has a nonvanishing component $u$ only in the axial direction, and $u$ is a function of position on a cross-section $\mathscr{Q}$ of the cylinder. Problems involving such deformations are ordinarily simpler than those in which the displacement vector has a more elaborate character, and for this reason they often serve usefully as pilot problems for the analysis of qualitative effects, especially when nonlinearity is involved. (Examples may be found in [1] and in the references cited in [2].) The present paper is intended to illustrate anti-plane shear in its role as exemplar in the setting of finite elasticity theory and with particular reference to an issue which has come to be called Ericksen's problem.

Prompted in part by the analytical and experimental work on large deformations of rubber by Rivlin and others, J. L. Ericksen raised the question of determining the class of all deformations which are possible according to the theory of finite elastostatics in every homogeneous, isotropic elastic material in the absence of body forces. (A presentation of the foundations of the theory of finite elasticity, including definitions of the terms homogeneous, isotropic, and body force, may be found in [3]. All materials considered in the present paper will be assumed to be homogeneous and isotropic.) A universal deformation of this kind can be maintained in each of two bodies identical in shape-but consisting of different elastic materials - solely by the application of suitably distributed forces to the surfaces of each body. (By imposing suitable body forces (as distinguished from forces applied to the surface) a given deformation can be brought about in any elastic material.) Quite apart from its theoretical interest, Ericksen's problem acquires additional significance because of its connection with the task of determining experimentally the stress-deformation relation characterizing the mechanical behavior of a given elastic material. A very brief account of this aspect of the matter may be found in the prefaces to Ericksen's articles as they appear reprinted in [4]. (See papers 10 and 11 in [4].)

In his first paper [5] on this subject, Ericksen posed the question for incompressible elastic materials - those such as rubber which admit only locally volume-preserving deformations. For materials of this kind, Ericksen's problem in its full generality is a rather difficult one, largely because of the complexity of the nonlinear system of partial differential equations involved. Although the problem was very nearly solved in full in [5], there remain some open questions which continue to attract attention. (See, for example, [6] and the references cited there.)

When the constraint of incompressibility is relinquished, the problem is easier and the class of universal deformations (which is smaller than in the incompressible case) was completely determined by Ericksen in [7]. A simpler and somewhat more direct proof of the main result established in [7] for the compressible case was given recently by Shield [8].

The miniature version of Ericksen's problem to be considered here may be roughly posed as follows: What is the most general finite anti-plane shear of a cylindrical body which is sustainable without body forces in every homogeneous, isotropic elastic material? Although the question is but a trivial special case of Ericksen's problem, it is analyzed here on an ad hoc basis rather than by appeal to the general results of [5] and [7]. The analysis illuminates clearly the distinction between compressible and incompressible materials and at the same time furnishes

J. K. Knowles received his Ph.D. in mathematics at MIT under Eric Reissner in 1957. Since 1958 he has been at California Institute of Technology, where he is now Professor of Applied Mechanics. His research has been in the theory of elasticity. - Editors 
an entertaining exercise in the elementary theory of analytic functions of a complex variable. The result has alternative interpretations in the theory of minimal surfaces as well as in the subject of steady flows of an inviscid fluid.

2. Finite anti-plane shear. Let $\mathscr{D}$ be an open, bounded, simply connected cross-section of a cylindrical region occupied by a homogeneous, isotropic elastic solid in its undeformed state. Take $x_{1}, x_{2}, x_{3}$ to be rectangular cartesian coordinates with the origin in $\mathscr{D}$ and the $x_{3}$-axis parallel to the generators of the cylinder. Suppose the body is deformed to an equilibrium state in which a particle originally located at $\left(x_{1}, x_{2}, x_{3}\right)$ moves to the point $\left(x_{1}, x_{2}, x_{3}+u\right)$, where $u=u\left(x_{1}, x_{2}\right)$ is the out-of-plane displacement associated with the anti-plane shear. It is shown in [2] that for such a deformation the field equations of finite elastostatics for an incompressible material can be reduced in the absence of body forces to the following system of three differential equations in two unknowns, $u$ and $q$ :

$$
\begin{gathered}
{\left[F\left(|\nabla u|^{2}\right) u_{, \beta}\right]_{, \beta}=0 \text { on } \mathscr{Q},} \\
q_{, \alpha}+\left[G\left(|\nabla u|^{2}\right) u_{, \alpha} u_{, \beta}\right]_{, \beta}=0 \text { on } \mathscr{D} .
\end{gathered}
$$

(The functions $q$ and $u$ are assumed to be once and twice continuously differentiable on $\mathscr{D}$, respectively.) In these equations, subscripts take the values 1,2 , a subscript preceded by a comma indicates partial differentiation with respect to the corresponding cartesian coordinate, and repeated subscripts are summed. Thus, for example,

$$
|\nabla u|^{2}=u_{, 1}^{2}+u_{, 2}^{2}=u_{, \beta} u_{, \beta} .
$$

The functions $F$ and $G$ of $|\nabla u|^{2}$ which appear in (2.1), (2.2) are given and depend on the particular material under consideration. ( $F$ and $G$ are related to the strain energy density-or elastic potential - associated with the material.) The unknown $u$ in (2.1), (2.2) is the out-ofplane displacement introduced above, while $q$ is related to the arbitrary hydrostatic pressure which occurs in the stress-deformation law because of the constraint of incompressibility. It should be emphasized that no linearization is involved in the derivation of (2.1), (2.2).

For a compressible elastic material, it is again possible in the case of anti-plane shear to reduce the field equations of the equilibrium theory of finite elasticity (this reduction is carried out in detail in [9]) to a system of the form (2.1), (2.2), except that now $q$ is related directly to $u$ by means of the additional equation

$$
q=H\left(|\nabla u|^{2}\right)
$$

where $H$ is a third given function characteristic of the material at hand.

The functions $F, G$ and $H$ are assumed to be continuously differentiable, and $F$ is required to be positive for all values of its argument.

There is no loss of generality involved in assuming, as will be done here, that

$$
u(0,0)=0 \text {, }
$$

since this may always be arranged by adding a constant, if necessary, to any solution $u$ of $(2.1)$, (2.2) or (2.1), (2.2), (2.3). This corresponds to the superposition of a rigid body translation in the axial direction.

In the incompressible case, the basic system (2.1), (2.2) consists of three differential equations in the two unknowns $u, q$; for compressible materials, the system (2.1), (2.2), (2.4) leads immediately to three differential equations for the single unknown $u$. The relevant set of equations is thus over-determined for either type of material, the more severe over-determination occurring in the compressible case. (This over-determination raises a question as to which, if any, elastic materials will actually sustain nontrivial states of anti-plane shear. This matter is studied in [2] for incompressible materials and in [9] for compressible ones.) To see that there are at least some solutions, one notes that in the incompressible case

$$
u=k_{\beta} x_{\beta}, \quad q=q_{0},
$$

where $k_{1}, k_{2}$, and $q_{0}$ are arbitrary constants, furnishes a solution of (2.1), (2.2) for any choice of 
$F$ and $G$ and hence for any incompressible material. An anti-plane shear in which $u$ has the form appearing in (2.5) is called a simple shear. Such a deformation subjects each cross-section $\mathscr{D}$ of the cylinder to a rotation about an axis in the plane of $\mathscr{D}$. The axis and the angle of rotation are determined by $k_{1}, k_{2}$ and are thus the same for all cross-sections.

For a compressible material, (2.6) also supplies a solution of (2.1), (2.2), (2.4), but now only if $q_{0}$ is chosen to be

so as to assure that (2.4) holds.

$$
q_{0}=H\left(k_{\beta} k_{\beta}\right)
$$

3. Universal states. The solution (2.6) furnishes a universal state of anti-plane shear for the incompressible case in the sense that it fulfills (2.1), (2.2) for every choice of $F, G$ and hence for all incompressible materials. The same may be said of the displacement field $u$ given by the first of (2.6) in the compressible case. The present section is devoted to the determination of all such universal states of finite anti-plane shear for each of the two classes of materials.

Suppose $u \neq 0$ is the out-of-plane displacement associated with any such universal state, for either the incompressible or the compressible case. Then $u$ is a universal solution of (2.1) in the sense that the latter equation is satisfied by $u$ for every positive, continuously differentiable function $F$. If in (2.1) the indicated differentiations are carried out, one finds

$$
F\left(|\nabla u|^{2}\right) u_{, \beta \beta}+2 F^{\prime}\left(|\nabla u|^{2}\right) u_{, \beta \gamma} u_{, \beta} u_{, \gamma}=0 \text { on } \mathscr{D},
$$

where the prime indicates differentiation. In particular (3.1) must hold for the special choice $F \equiv 1$, so that $u$ must satisfy Laplace's equation:

$$
u_{, \beta \beta}=0 \text { on } \mathscr{D} \text {. }
$$

(This choice of $F$ corresponds in the incompressible case to the so-called Mooney-Rivlin model for the mechanical behavior of certain rubberlike materials. See chapter 10 of [10].) On the other hand, for any material for which $F^{\prime}(t) \neq 0$ for all $t \geqslant 0,(3.1)$ and (3.2) show that $u$ must also satisfy

$$
u_{, \beta \gamma} u_{, \beta} u_{, \gamma}=0 \text { on } \mathscr{D} \text {. }
$$

Thus (3.2), (3.3) are necessary if $u$ is to be a universal solution of (2.1). It is clear from (3.1) that they are also sufficient.

In order to determine all functions $u$ which are twice continuously differentiable on $\mathscr{D}$ and satisfy (3.2) and (3.3), set $z=x_{1}+i x_{2}$ and let $f(z)$ be the function analytic on $\mathscr{D}$ whose real part is the harmonic function $u$ and for which

$$
f(0)=0 \text {. }
$$

(Recall from $(2.5)$ that $u(0,0)=0$.) It is easy to show that $(3.3)$ is then equivalent to

$$
\operatorname{Re}\left\{\left[f^{\prime}(z)\right]^{2} \overline{f^{\prime \prime}(z)}\right\}=0, \quad z \text { in } \mathscr{D},
$$

where the bar denotes complex conjugate and Re stands for real part. It is not possible for $f^{\prime}(z)$ to vanish identically on $\mathscr{D}$; if it did, (3.4) would lead to $f \equiv 0$ and hence to $u \equiv 0$. As a result, there are a point $z_{1}$ in $\mathscr{D}$ and a neighborhood $\mathscr{N}$ of $z_{1}$ lying entirely in $\mathscr{Q}$ such that $f^{\prime}(z) \neq 0$ for $z$ in $\mathscr{N}$. One may therefore define

$$
g(z)=\frac{1}{f^{\prime}(z)}, \quad z \text { in } \Re ;
$$

$g$ is analytic on $\Re$. Direct calculation shows that (3.5) is equivalent to

$$
\operatorname{Re}\left[g^{\prime}(z)\right]=0, \quad z \text { in } \Re \text {. }
$$

It follows from the Cauchy-Riemann equations applied to $g^{\prime}$ that

$$
g^{\prime}(z)=i c, \quad z \text { in } \Re
$$

where $c$ is a real constant.

It is now useful to distinguish two cases: $c=0$ and $c \neq 0$. Suppose first that $c=0$. Then (3.8) implies that $g$ is constant on $\Re$, say 


$$
g(z)=\frac{1}{k}, \quad z \text { in } \Re, \quad k=k_{1}-i k_{2}
$$

where $k_{1}$ and $k_{2}$ are real constants. From (3.9), (3.6) one concludes that $f^{\prime}(z)=k$ for $z$ in $\Re$ and hence, by the identity theorem for analytic functions, that $f^{\prime}(z)=k$ for all $z$ in $\mathscr{D}$ as well. Thus

$$
f(z)=k z, \quad z \text { in } \mathscr{D} \text {. }
$$

Next, suppose that $c \neq 0$ in (3.8). Then

$$
g(z)=i c\left(z-z_{0}\right), \quad z \text { in } \Re,
$$

where $z_{0}$ is an arbitrary complex constant. Since, by (3.6) and the analyticity of $f, g$ cannot vanish in $\Re$, one concludes that $z_{0}$ lies outside $\Re$. Substitution from (3.11) into (3.6) leads to

$$
f(z)=\frac{1}{i c} \log \left(z-z_{0}\right)+d, \quad z \text { in } \Re
$$

where $d$ is a constant of integration. Here the logarithm is to be single-valued and analytic in the $z$-plane cut along a ray from $z_{0}$ to infinity. Since $f$ must be analytic in all of $\mathscr{D}$, another argument based on the identity theorem shows that $z_{0}$, as well as the cut, must in fact lie outside of $\mathscr{D}$. (Note that the simple connectivity of $\mathscr{D}$ plays a role here.) Finally, invoking the normalization (3.4) one has

$$
f(z)=\frac{1}{i c}\left[\log \left(z-z_{0}\right)-\log \left(-z_{0}\right)\right], \quad z \text { in } \mathscr{D} .
$$

Collecting the results (3.10) and (3.13) and taking real parts provides two families of universal solutions of (2.1):

$$
\begin{gathered}
u=k_{\beta} x_{\beta} \\
u=\frac{1}{c}\left(\theta-\theta_{0}\right), \quad c \neq 0,
\end{gathered}
$$

where

$$
\theta=\theta\left(x_{1}, x_{2}\right)=\arg \left(z-z_{0}\right), \quad \theta_{0}=\arg \left(-z_{0}\right) .
$$

The solution (3.14) is the simple shear which appears in (2.6). Equation (3.15), however, describes a more complicated deformation in which the collection of particles which occupies the domain $(2)$ before deformation is carried into a portion of a right helicoid of pitch $2 \pi / c$ whose axis passes through $z_{0}$ and is parallel to the generators of the cylinder. (To see quickly that (3.15) indeed supplies a universal solution of (2.1), one need only write (2.1) in polar coordinates centered at $z_{0}$.)

It was observed in the preceding section that the simple shear (3.14) is a universal state of anti-plane shear for either incompressible or compressible materials. To determine the status in this respect of the second universal solution of (2.1), it is necessary to see whether $u$ as given by (3.15) survives when subjected to the additional restriction (2.2) in the incompressible case or to (2.2), (2.4) in the compressible case. To this end, suppose that $u$ is any universal solution of (2.1), so that (3.2) and (3.3) hold. It is easy to show that (2.2) may then be written in the form

$$
\left[q+K\left(|\nabla u|^{2}\right)\right]_{, \alpha}=0 \text { on } \mathscr{D} \text {, }
$$

where

$$
K(t)=\frac{1}{2} \int_{0}^{t} G(\tau) d \tau, \quad t \geqslant 0 .
$$

It follows immediately that any universal solution of (2.1) will supply a universal state of anti-plane shear for incompressible materials, since it is possible to choose $q=-K\left(|\nabla u|^{2}\right)$ and thus to satisfy (2.2). One concludes that $u=u\left(x_{1}, x_{2}\right)$ corresponds to a universal state of finite anti-plane shear for homogeneous, isotropic, incompressible elastic materials if and only if it is either a simple shear (3.14) or a deformation of the form (3.15) which carries a typical cross-section of the cylinder into a portion of a right helicoid.

For compressible materials, on the other hand, a universal solution of (2.1) must also satisfy 
(2.4) as well as (3.17) if it is to furnish a universal state of anti-plane shear. This means that $u$ must be such that

$$
H\left(|\nabla u|^{2}\right)+K\left(|\nabla u|^{2}\right)=\text { constant on } \mathscr{D}
$$

for every choice of the functions $H$ and $G$. Choosing $H(t) \equiv 1, G(t) \equiv 1$, corresponding to $K(t)=\frac{1}{2} t$, one finds from (3.19) that $|\nabla u|$ is necessarily constant on $\mathscr{D}$. While this is of course true for $u$ as given by (3.14), it fails when $u$ is of the form (3.15). As a consequence, the only universal state of finite anti-plane shear for homogeneous, isotropic, compressible elastic materials is the simple shear (3.14).

These results are indeed special cases of the universal deformations found by Ericksen in [5] and [7].

4. Other interpretations. The fact that all universal solutions of (2.1) are given by (3.14) and (3.15) has simple interpretations in areas other than finite elastostatics. If, for example, $F$ is chosen to be

$$
F\left(|\nabla u|^{2}\right)=\left(1+|\nabla u|^{2}\right)^{-\frac{1}{2}},
$$

then (2.1) reduces to the differential equation for minimal surfaces (see [11], pp. 182-187) when the latter are represented in the form $x_{3}=u\left(x_{1}, x_{2}\right)$. The results of the preceding section show that the only minimal surfaces for which $u$ is a harmonic function of $x_{1}, x_{2}$ are the plane and the right helicoid.

Still another interpretation of the universal solutions (3.14), (3.15) may be given in terms of fluid flow. The field equations for plane, irrotational, steady flow of an inviscid fluid may be reduced (see [12] chapter 1) to a single equation of the form (2.1), where $u$ is the velocity potential, $\nabla u$ the fluid particle velocity, and $F\left(|\nabla u|^{2}\right)$ the fluid density at a particle whose speed is $|\nabla u|$. The special case $F \equiv$ constant corresponds to an incompressible fluid, for which the velocity potential is a harmonic function. The universal solutions (3.14), (3.15) thus describe the only plane, steady, irrotational flows which are possible for both compressible and incompressible inviscid fluids. The flow associated with (3.14) is uniform with a velocity vector whose component in the $x_{\alpha}$-direction is $k_{\alpha}$. A velocity potential of the form (3.15) corresponds to a vortex flow with constant angular speed $1 / c$ about an axis through the point $z_{0}$ and perpendicular to the $x_{1}, x_{2}$-plane.

\section{References}

1. J. K. Knowles, The finite anti-plane shear field near the tip of a crack for a class of incompressible elastic $\mathrm{n} \rightarrow$ rials, International Journal of Fracture, 13 (1977) 611-631.

2. , On finite anti-plane shear for incompressible elastic materials, J. Austral. Math. Soc., Series B, 19 $(\Im$ ( $400-415$.

3. C. Truesdell, The Elements of Continuum Mechanics, Springer-Verlag, New York, 1966.

4. _ ed., Continuum Mechanics IV, Problems in Non-Linear Elasticity, Gordon and Breach, New York, 1965.

5. J. L. Ericksen, Deformations possible in every isotropic, incompressible, perfectly elastic body, Z. Angew. $\Lambda \rightarrow$ i. Phys., 5 (1954) 466-489.

6. S. E. Martin and D. E. Carlson, A note on Ericksen's problem, J. of Elasticity, 6 (1976) 105-108.

$\rightarrow 7$. J. L. Ericksen, Deformations possible in every compressible, perfectly elastic material, J. Math. Phys., 34 (1955) $126-128$.

8. R. T. Shield, Deformations possible in every compressible, perfectly elastic material, J. of Elasticity, 1 (: $\leadsto$ ) 91-92.

9. J. K. Knowles, A note on anti-plane shear for compressible materials in finite elastostatics, J. Austral. $\Lambda \rightarrow$. Soc., Series B, 20 (1977) 1-7.

10. A. E. Green and J. E. Adkins, Large Elastic Deformations, Clarendon, Oxford, 1960.

11. D. J. Struik, Lectures on Classical Differential Geometry, Addison-Wesley, Cambridge, 1950.

12. L. Bers, Mathematical aspects of subsonic and transonic gas dynamics, Surveys of Applied Mathematics, vol. 3, Wiley, New York, 1958.

Division of Engineering and Applied Science 104-44, California Institute of Technology, Pasadena, CA 91125. 